\title{
Influence of temperature and humidity on bakelite resistivity
}

\author{
R.Arnaldi ${ }^{\mathrm{a}}$, A.Baldit ${ }^{\mathrm{b}}$, V.Barret ${ }^{\mathrm{b}}$, N.Bastid ${ }^{\mathrm{b}}$, G.Blanchard ${ }^{\mathrm{b}}$,

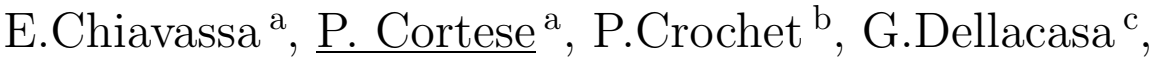 \\ N.De Marco ${ }^{\text {a }}$, P.Dupieux ${ }^{\mathrm{b}}$, B.Espagnon ${ }^{\mathrm{b}}$, J.Fargeix ${ }^{\mathrm{b}}$, \\ A.Ferretti $^{\mathrm{a}}$, M.Gallio $^{\mathrm{a}}$, L.Lamoine $^{\mathrm{b}}$, L.Luquin $^{\mathrm{d}}$, F.Manso $^{\mathrm{b}}$, \\ V.Metivier $^{\mathrm{d}}$, A.Musso $^{\mathrm{a}}$, C.Oppedisano ${ }^{\mathrm{a}}$, A.Piccotti ${ }^{\mathrm{a}}$, \\ A.Rahmani ${ }^{\mathrm{d}}$, L.Royer ${ }^{\mathrm{b}}$, O.Roig ${ }^{\mathrm{b}}$, E.Scalas ${ }^{\mathrm{c}}$, E.Scomparin ${ }^{\mathrm{a}}$, \\ E. Vercellin ${ }^{\text {a }}$
}

\section{For the ALICE collaboration}

\begin{abstract}
${ }^{a}$ Sezione INFN di Torino and Dipartimento di Fisica Sperimentale dell'Università, Via Pietro Giuria 1, 10125 Torino, Italy

${ }^{\mathrm{b}}$ LPC Clermont Ferrand - IN2P3/CNRS and Univ. Blaise Pascal, 63177 Aubiere Cedex, France

${ }^{\mathrm{c}}$ Universita del Piemonte Orientale, Dipartimento Scienze e Technologie Avanzate, Corso Borsalino 54, 15100 Alessandria, Italy

${ }^{\mathrm{d}}$ Ecole des Mines de Nantes, Subatech nantes, 4 Rue Alfred Kastler, 44070 Nantes Cedex 03, France
\end{abstract}

\begin{abstract}
The use of phenolic or melaminic bakelite as RPC electrodes is widespread. The electrode resistivity is an important parameter for the RPC performance. As recent studies pointed out, the bakelite resistivity changes with temperature and is influenced by humidity. In order to gain a quantitative understanding on the influence of temperature and humidity on RPC electrodes, we assembled an apparatus to measure resistivity in well-controlled conditions. A detailed description of the experimental set-up as well as the first resistivity measurements for various laminates in different environmental conditions are presented.
\end{abstract}

Key words: Bakelite; resistivity; temperature; humidity 


\section{Introduction}

Recent studies have shown that the performance (in particular the rate capability) of RPCs strongly depends on the bulk resistivity, $\rho$, of the electrodes $(1),(2),(3)$.

One of the most widespread electrode is the bakelite plate. Since the bulk resistivity of bakelite changes as a function of temperature and humidity, we have set up a measuring apparatus in order to carefully investigate this dependence; this study was performed in the framework of the dimuon trigger for the ALICE experiment.

The quality of the interface contact between the resistance meter electrodes and the bakelite plane is of special importance in these measurements. We have therfore tried various techniques: the conductive-gel, conductive-rubber, and deposited-metal methods in order to investigate the possible presence of systematic errors. The metal used in the last method is a few $\mu \mathrm{m}$ thick silver layer deposited in vacuum on the bakelite surface.

Different bakelite types may have rather different behaviours, for this reason we started a systematic analysis. Here we will present preliminary results about a phenolic plate and a melaminic bakelite plate.

\section{Experimental method}

In Fig. 1a, a sketch of the experimental set-up is shown. The bakelite plate is conditioned in a climatic cell (Challenge $500-\mathrm{V}$ by Angelantoni S.p.A.) able to control temperatures in the range $-20 /+70{ }^{\circ} \mathrm{C}$ and relative humidities in the range $25-95 \%$ for temperatures between 10 and $70{ }^{\circ} \mathrm{C}$. The temperature and humidity accuracy are respectively $1^{\circ} \mathrm{C}$ and $5 \%$. The internal volume is $6.9^{*} 10.6^{*} 19.6 \mathrm{dm}^{3}$. Two brass electrodes are in contact with the two sides of the bakelite plate and are connected to a high resistance meter (HP 4339 B, Hewlett Packard) which can supply a voltage between 0.1 and 1000 V D.C. and measure resistances between $10^{3} \Omega$ and $1.610^{16} \Omega$ with an accuracy of $0.6 \%$. The resistance meter is computer controlled via a GP-IB interface card by a PC running LabView ${ }^{\mathrm{TM}}$.

A direct comparison at room temperature between the deposited-metal and the conductive-gel methods gives nearly the same results. The resistivity measured in the same conditions with the conductive-rubber method depends on the pressure applied on the electrodes as can be seen from Fig 1b. The resistivity decreases with pressure and stabilizes to a value which is nearly the double of that measured by the other two methods.

In Fig. 2, we show a comparison among the three different contact methods listed above. The resistivity of the phenolic plate is plotted as a function of temperature for three different relative humidity values: 30, 40, $50 \%$. The 


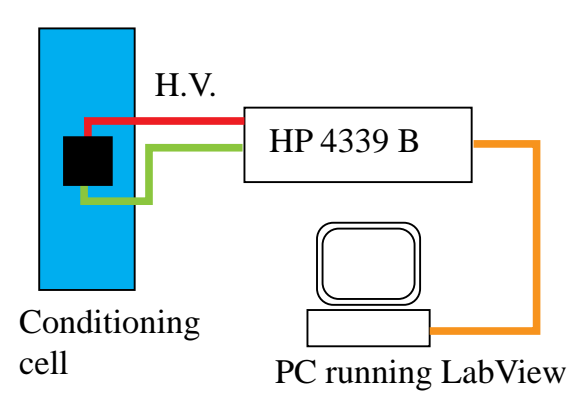

(a)

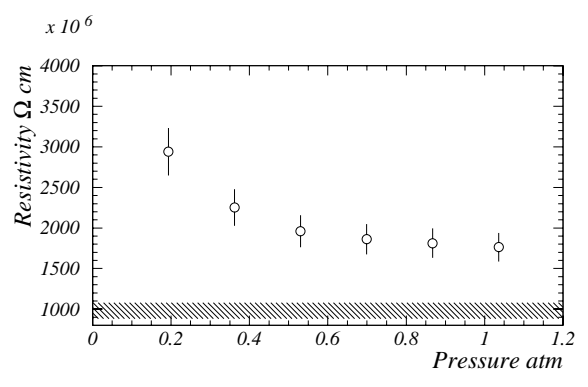

(b)

Fig. 1. (a) Sketch of the experimental set-up, (b) Pressure dependence of resistivity measurement by the conductive-rubber method on the phenolic plate (dots); the shaded band represents the values for the other methods

phenolic plate has been conditioned from 1 to 3 days to set the humidity and from 1 to 24 hours to set the temperature with no significant difference in the results. For the conductive-rubber technique and metal-deposition technique, measurements have been performed simultaneously on two different samples of the same bakelite plate. The resistance values have been measured with the bakelite inside the chamber for all the investigated temperatures. The results show the same trend in both cases - the resistivity slightly decreases with humidity. The temperature dependence is stronger and the resistivity drops exponentially. There is a systematic scale factor among the two measurements due to the different contact type (the applied pressure on the rubber electrodes is $\simeq 1 \mathrm{~atm}$ ).

The resistivity measured with the gel technique has a different temperature trend. Indeed, to prevent gel drying during conditioning, the phenolic plate has been removed from the chamber just before gel deposition and resistance measurement. Successive measurements have shown that the reliability of this procedure is limited since the temperature of the bakelite very rapidly reaches the one of the environment. For the melamine sample in Fig. 3 we show the measured temperature dependence obtained by the silver electrode method.

\section{Conclusions and outlook}

In this paper we have shown that it is advisable to measure the resistivity of bakelite in well controlled temperature and humidity conditions. The results obtained by the conductive rubber method depend on the applied pressure and, for the highest pressure we reached, they are higher by a factor of two than those measured by the other methods. Finally there is an evident dependence of resistivity on temperature in the investigated interval. The resistivity decreases by a factor of ten within a $20{ }^{\circ} \mathrm{C}$ interval. Long conditioning times 

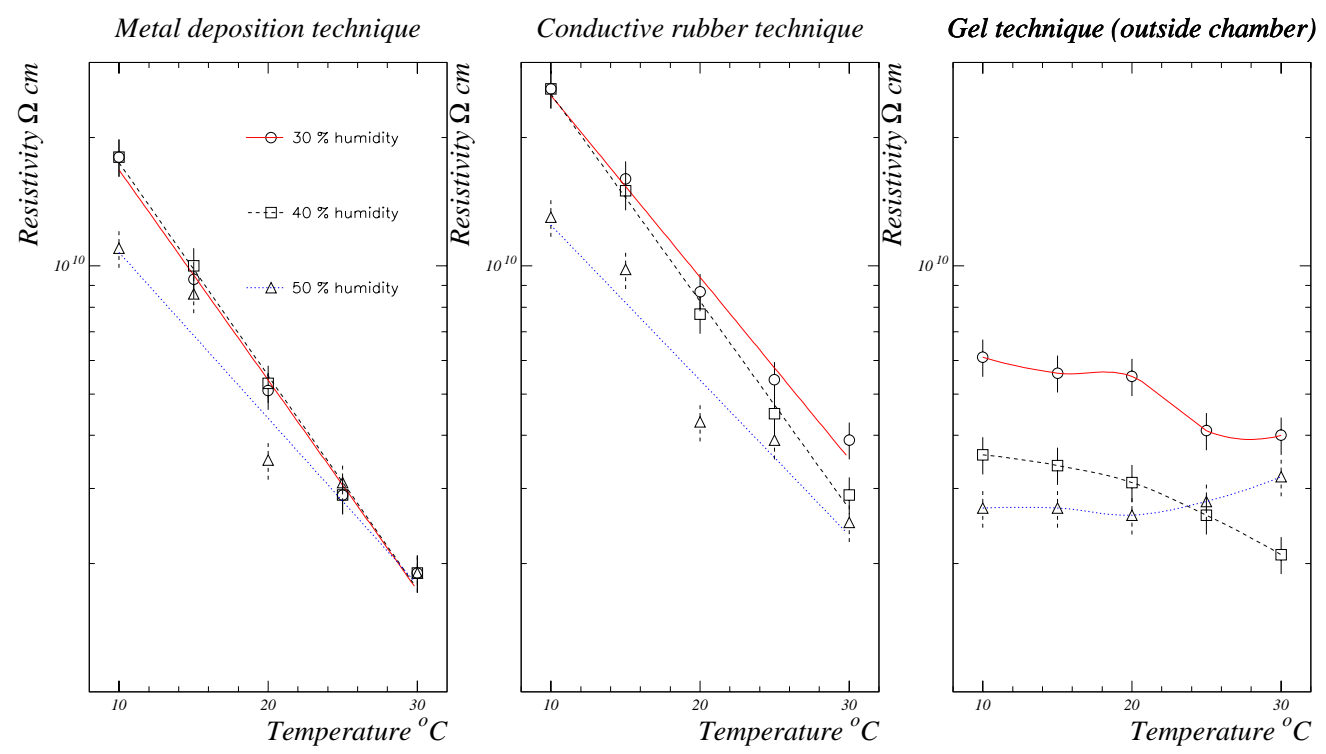

Fig. 2. Preliminary results for resistivity of phenolic bakelite measured by three different methods

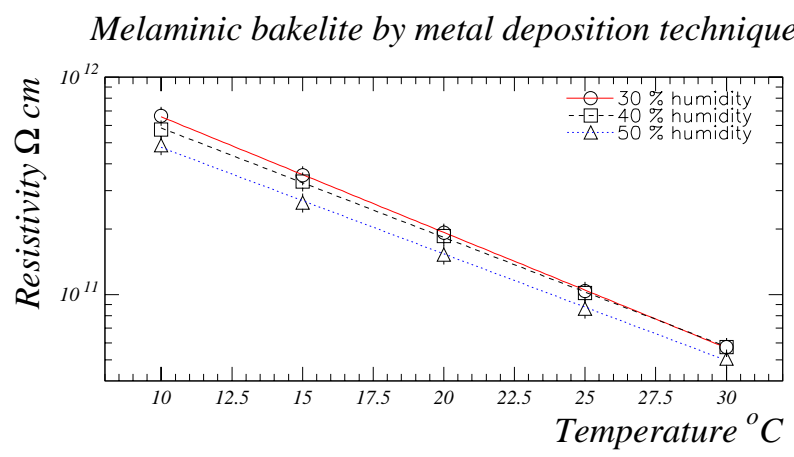

Fig. 3. Preliminary results for resistivity measurement of melaminic bakelite by the deposited metal method

will be necessary to properly understand the influence of the humidity.

Among the future experiments we plan to study the effect of linseed oil and resistivity memory effects after heating at more than $60^{\circ} \mathrm{C}$.

We would like to thank P. Vitulo for stimulating discussion. Many thanks are due to P.F. Ottria for his valuable technical support.

\section{References}

[1] Y. Inoue et al., Nucl. Instr. and Methods A 372 (1996) 39.

[2] R. Arnaldi et al., to be published in Quark Matter 99 Proceedings

[3] R. Arnaldi et al., published in the Proceedings of this Workshop 